\title{
TWO-PHASE COOLING FLOWS WITH MAGNETIC RECONNECTION
}

\author{
Colin Norman ${ }^{1}$ \\ and \\ Avery Meiksin ${ }^{2}$ \\ Institute for Theoretical Physics, University of California, Santa Barbara \\ Received __; accepted _
}

To appear in The Astrophysical Journal (1 September 1996)

1 Johns Hopkins University, Department of Physics and Astronomy, 3400 N. Charles Str., Baltimore, MD 21218; norman@stsci.edu

${ }^{2}$ University of Chicago, Department of Astronomy and Astrophysics, 5640 S. Ellis Ave., Chicago, IL 60637; Edwin Hubble Research Scientist; meiksin@oddjob.uchicago.edu 


\begin{abstract}
Motivated by the observations of high Faraday rotation measures measured in cooling flow clusters we propose a model relevant to plasmas with comparable thermal and magnetic pressures. Magnetic field reconnection may play a major role in changing the topology of the magnetic field in the central cooling flow regions. The effect of the topology change is that cool flux loops can reconnect to hot flux loops that are connected to the overall thermal reservoir of the cluster. There can be a rapid recycling of mass between hot and cold phases on a time scale of $\sim 3 \times 10^{8}-10^{9} \mathrm{yr}$ which may reduce the inferred inflow and mass condensation rates by at least an order of magnitude. A central multiphase medium is a direct consequence of such a model. Throughout the cooling flow the filling factor of the hot loops $\left(T>2 \times 10^{7} \mathrm{~K}\right)$ is of order unity. The filling factor of the cool $\operatorname{loops}\left(<2 \times 10^{7} \mathrm{~K}\right)$ is $0.1-1 \%$ with a corresponding mass fraction of cold phase of $1-10 \%$. A crucial parameter is the coherence length of the field relative to the cooling radius and the distribution of field energy with scale. When the cooling radius is greater than the field coherence length then cooling flows proceed as usual. When the coherence length is greater than the central cooling radius, however, the thermal energy of the reservoir can be tapped and the mass condensation rates may be very significantly reduced. Three additional conditions must be satisfied: I. Cold loops must be able to fall at least as far as the mean distance between hot loops in a cooling time; II. Loops must enter an evaporative phase on reconnecting; and III. A sufficient number of hot loops penetrate the cold phase region to power the radiative losses.
\end{abstract}




\section{INTRODUCTION}

Calculation of the mass flow rate into the centers of clusters of galaxies with short cooling times gives large values of order $\sim 100-1000 M_{\odot} \mathrm{yr}^{-1}$ (Binney and Cowie 1981). This point has dominated most of the thinking on the thermal evolution and central gas dynamics of clusters of galaxies (Fabian 1994). We propose a mechanism that will reduce these mass inflow rates by an order of magnitude in clusters where the coherence length of the field is greater than the cooling radius. The mechanism we invoke here involves, however, the study of turbulent magnetized fluids. Given our state of knowledge of the properties of these flows our calculations can, at present, only be regarded as indicative. However, the stabilization does seem to be a reasonable mechanism and we present it here in the spirit of the pioneering feedback work of Tabor and Binney (1993) and Binney and Tabor (1995).

Our motivation for rethinking this subject was the beautiful data from Ge (1991), Ge and Owens (1993), Owens et al. (1990) clearly showing that cooling flow clusters have rotation measures between 10-100 times those that have no established cooling. The inferred field strengths are at or near equipartition values with the thermal pressure. Magnetized clusters have been studied before in detail by Soker and Sarazin (1990). They assumed that once the magnetic field built up it was annihilated down to equipartition values via reconnection process. Normally, however, reconnection does not annihilate magnetic fields (Taylor 1974). Even with fast reconnection, annihilation of significant volumes of magnetic energy density does not take place.

To dissipate the field globally requires a global anomalous resistivity and this is very difficult both to achieve and sustain except in very small volumes.

The issues we have to address are complex. Magnetic field reconnection normally rearranges the field and may allow some change in the mass-to-flux ratio of loops. Since the magnitude of the field does not change significantly during reconnection the magnetic pressure is not significantly altered. The crucial point of physics for this work, however, is that reconnection significantly alters the topology and connectedness of the magnetic field. 
In the central regions of cooling flows the line widths are random (Heckman et al. 1989) and it is most likely that the fluid is turbulent. The most recent analysis of the nature of thermal conduction in cooling flows (Tao 1996) indicates that there may be some but not complete suppression of the thermal conductivity. Additional suppression may result from electromagnetic instabilities in the cluster gas (Pistinner et al. 1996). We will adopt the values consistent with Tao's work in our subsequent calculations although we regard the thermal conductivity in a tangled field as still an unsettled question (cf. Rosner and Tucker 1989, Tribble 1989, Chun and Rosner 1993).

Recent spectral X-ray measurements have established that the X-ray emitting gas is composed of two (or more) phases (Fukazawa et al. 1994; Fabian et al. 1994). We incorporate this important new datum into our model. Our proposed solution to the suppression of the large mass drop out rates in cooling flows is to recycle the cooling $\left(\leq 10^{7} \mathrm{~K}\right)$ gas back into the hot phase $\left(\geq 10^{7} \mathrm{~K}\right)$ and then allow it to cool again. Only a small fraction of the total cooling mass need be in the cool phase to match the observed low-temperature X-ray emission. The mass condensation rate is, then, the amount of cool gas per recycling time. Since current estimates of the amount of condensed mass are calculated assuming that the cooling gas eventually leaves the flow with no recycling, the currently estimated masses can be suppressed by the ratio of the recycling time to the age of the cluster.

More specifically, the recycling solution we propose is as follows:

The magnetic field lines engulfed by the outward moving cooling wave have a spectrum of scales. The ones with scales greater than the scale of the cooling wave remain connected to the essentially infinite heat reservoir of the cluster. Even with the turbulent thermal conductivity given by Tao (1996), field lines topologically connected to the outside of the cooling wave region remain hot and can be treated as isothermal. These hot tubes are the pipes that the cooling flux loops can tap into. Smaller scale cooling loops are not connected to the heat bath of the cluster. However, small-scale cooling loops can be heated and recycled by the process of magnetic field reconnection giving the required recycling mechanism. 
In the next section (2) we set up a simple physical model and present estimates of the characteristics of our solution. Thermal structure and recycling are discussed in more detail in section 3. In Section 4 we discuss some observational consequences and a summary and conclusions are given.

\section{BASIC MODEL AND ESTIMATES}

Assume an initially tangled isotropic field across the cluster with a plasma parameter $\beta=\left(P_{\text {thermal }} / P_{\text {magnetic }}\right)=\beta_{0} \geq 10$ since the cluster is initially dominated by thermal pressure and not magnetic pressure. We assume there is a spectrum of loops as a function of scale. We envisage these intracluster fields to be generated by radio galaxies, outflows from AGNs and starburst galaxies and the outflow processes from normal galaxies that enrich the intracluster medium that are presumably from a supernovae driven source. With these processes in mind we estimate that the scales of the fields in the general intracluster medium can range from $\sim 10 \mathrm{kpc}$ up to $\sim 1 \mathrm{Mpc}$. The field may be completely coherent across the cluster if there is a large scale primordial field or if large scale fields are generated in the process of structure formation (Kulsrud 1995).

After a time $t$ the material enveloped by the outwardly propagating cooling wave (with radius defined by that radius at which $t_{\text {cool }}=t$ ) can cool and move inward (Bertschinger 1989). In the kinematic inflow phase the fields are frozen in and the stretching and amplification that occur are well described in the paper by Soker and Sarazin (1990). The field becomes increasingly radial and we will assume for simplicity of analysis that it is exactly radial in this phase.

Assuming complete ionization, the cooling law that describes the propagation of the cooling wave is given by:

$$
\frac{3}{2} \frac{d n k T}{d t}=n^{2} \Lambda(T)
$$

where $T$ is the gas temperature, $n$ is the density, and $\Lambda(T)$ is the cooling function. We assume that the gas and dark matter both have the distribution of an isothermal sphere with a gas 
temperature, $T_{g}$, and dark matter temperature, $T_{d}$, with gas density

$$
n=n_{0}\left(\frac{r_{0}}{r}\right)^{\alpha}
$$

where $\alpha=2 T_{d} / T_{g}$ and the subscript 0 refers to gas properties at a fiducial radius $r_{0}$. The outwardly propagating cooling wave has the solution:

$$
r_{\mathrm{cool}}=r_{0}\left(\frac{t}{t_{\mathrm{cool}, 0}}\right)^{1 / \alpha},
$$

with

$$
v_{\mathrm{cool}}=v_{\mathrm{cool}, 0}\left(\frac{r_{0}}{r_{\mathrm{cool}}}\right)^{\alpha-1}
$$

where

$$
t_{\mathrm{cool}, 0}=\left[\frac{3 k T_{0}}{2 n_{0} \Lambda\left(T_{0}\right)}\right]
$$

and $v_{\mathrm{cool}, 0}=\left(r_{0} / t_{\mathrm{cool}, 0}\right)$. The mass flux into the cooling bubble, at any given time $t$ and at radius $r_{\text {cool }}$, is:

$$
\dot{M}_{\text {cool }}=\dot{M}_{0}\left(\frac{r_{\text {cool }}}{r_{0}}\right)^{3-2 \alpha}
$$

where

$$
\dot{M}_{0}=\left(\frac{4 \pi \rho_{0} r_{0}^{3}}{t_{\mathrm{cool}, 0}}\right) .
$$

As the cooling wave propagates outward, the material trapped in small magnetic loops will start to cool and fall inward.

The plasma parameter $\beta$ varies as a function of radius as:

$$
\beta=\beta_{0}\left(\frac{r}{r_{0}}\right)^{\alpha / 3}
$$

and the flow can be significantly magnetized when $\beta \sim 1$. We are assuming that the flow is sufficiently large as to entrain field lines into the central regions of the cooling flow, allowing a build up of the magnetic pressure. The increased magnetic pressure does not change the estimates of the accretion rates that are based purely on the thermal properties, not the momentum balance.

As discussed by Bertschinger and Meiksin (1986) and Meiksin (1988), if the thermal conductivity is within an order of magnitude of the Spitzer value, the cooling along flux tubes that 
are simply connected to the outside of the cooling wave is negligible. Therefore these connected flux tubes do not contain any cool material. The flux tubes that are not connected can cool. This leads to a two-phase medium of hot connected flux tubes and cool disconnected tubes. As discussed by Soker and Sarazin (1990) even if all the flux were reconnected and dissipated the associated heat input would have negligible effect on the thermal balance.

Reconnection is a natural way to recycle the cold material back into the hot phase. The exact details are unclear but the result is probably bounded by the rules for collisions of flux tubes (cf. Norman et al. 1996) as a lower bound or explosive reconnection with all the mass injected into the background medium between hot and cold phases as an upper bound.

Let us assume that a fraction, $f_{h}$, of the mass and flux is in hot tubes and the rest in cold tubes. Let us also assume that there is a mean flux reconnection rate, $\Gamma$, giving a mean mass exchange rate $\dot{M}_{e}$ between hot and cold of:

$$
\dot{M}_{e}=\left(\frac{\Phi}{M}\right)^{-1} \Gamma \Phi
$$

where the flux to mass ratio must be taken in mean averaged sense here. Therefore since the timescale for mass exchange is $\Gamma^{-1}$, the fraction of the total mass, $M_{h}$, within a cooling radius that is in the form of cool condenstates $\left(<10^{7} K\right)$ is:

$$
M_{\text {rec }}=(\Gamma \tau)^{-1} M_{h},
$$

where $\tau$ is the time for mass to leave the hot phase, which is on the order of the age of the cluster because of thermal heat conduction. The amount of mass being recycled at any given time is negligible compared to the total amount of mass in the hot phase.

Concentrating on one loop and using the fast reconnection law, Soker and Sarazin (1990) give a typical reconnection timescale, $\tau_{R}=\Gamma^{-1} \sim l_{t} /\left(\epsilon V_{A}\right)$ of:

$$
\tau_{R}=10^{8} \mathrm{yr}\left(\frac{\epsilon}{0.1}\right)^{-1}\left(\frac{l_{t}}{3 \mathrm{kpc}}\right)\left(\frac{B}{100 \mu G}\right)^{-1}\left(\frac{n}{0.03 \mathrm{~cm}^{-3}}\right)^{\frac{1}{2}} .
$$

for our parameters. We assume that reconnection occurs with a reconnection velocity of $\epsilon$ times the Alfven velocity. The characteristic scale length for reconnection is here taken to be the 
transverse dimesion of a loop. Note that when large scale hot loops reconnect with small-scale cool loops the reconnection time given in (12) should be regarded as an upper limit since the reconnection time may be considerably shorter due to the smaller size of the loops. The internal Alfven speed $V_{A}$ in the cool and hot loops does not change significantly since $V_{A} \propto n^{\gamma}$ where $0 \leq \gamma \leq 1 / 6$. Complete reconnection will occur on a single collision if the reconnection time is less than or equal to the crossing time of the loops. If this is not satisfied then collisions with hot loops will continue until the process is complete. This is similar to shredding. For more details regarding incomplete reconnection during flux loop collisions see Norman et al. (1996). Generally, the complex process of shredding of loops and blobs as they fall into the central regions of the cooling flow is one where cool gas distribution cascades to smaller and smaller scales. A small inefficiency in the recycling would permit some material to continue to cool and make stars, but at a much reduced rate compared to the standard cooling flow prediction.

We note here that buoyancy is an inefficient means for transporting flux out of the cooling region (Soker and Sarazin 1990). Flux loops will be buoyant and reach an appropriate scale height. We assume that most of the loops are still trapped, although there is probably still some flux loss. Reconnection will then occur and the value of mass and flux for the product loops will change. There will then be a rising and falling of loops as the reconnection process continues and re-sorts the mass and flux, although it is not possible to follow an individual piece of flux and mass. Magnetic tension in reconnected loops may lift the cool optically-emitting material out of the center of a cooling flow, accounting for the wide range of scales over which the optical filaments are observed (Zoabi et al. 1996). In practice, loops will shred into smaller structures due to Kelvin-Helmholtz instabilities after only a few Brunt-Väsäilä oscillation periods (Reale et al 1991). As discussed below this will enhance their evaporation and mass recycling.

\section{THERMAL STRUCTURE AND RECYCLING}


We now describe the thermal structure of the intracluster gas. The radiative cooling of the hot phase is nearly balanced by heat conduction from the outer portions of the cluster into the cooling region by those extended flux tubes that connect the external heat reservoir to the cooling interior. While perfect balance between cooling and heat conduction requires too high an external temperature compared to observations, acceptable solutions are found when a small amount of inflow is allowed (Bertschinger \& Meiksin 1986), provided the temperature decline is not more than by a factor of $\sim 2-3$ (Meiksin 1988). Accordingly, we may expand the fluid variables in the small parameter $\epsilon=\dot{M}_{\text {flow }} / \dot{M}_{h}$, where $\dot{M}_{\text {flow }}$ is the true accretion rate, and $\dot{M}_{h}$, given by equation (7) above and evaluated at the cooling radius, is the rate that would be inferred from the $\mathrm{X}$-ray luminosity assuming heat conduction were suppressed. Thus, for example, we may write, to first order, $n(r)=n_{0}\left(r / r_{\text {cool }}\right)^{-\alpha}\left[1+\epsilon n_{1}(r)\right]$ and $T_{h}(r)=T_{0}(r)\left[1+\epsilon T_{1}(r)\right]$. The zeroth order equation for pressure support (both thermal and magnetic) then requires, for $\beta>1$,

$$
\alpha=\frac{2 T_{d}}{T_{h}}\left[1+\beta(r)^{-1}\right]^{-1}
$$

when the gas temperature varies slowly with radius compared to the density. We point out that in the case $T_{d} \sim T_{h}$ and where the cooling flow is sufficiently magnetized with $\beta \sim 1$, then $\alpha \sim 1$. Consequently, from equation (6), $\dot{M}_{\text {cool }} \propto r$, a case favored by the observations. We will regard $\alpha$ below as being directly determined from the measurements of the X-ray surface brightness of the hot component, since the radial dependence of the surface brightness depends only weakly on that of the temperature.

Setting $x=r / r_{\text {cool }}$ and $y=T_{0}(r) / T_{h}$, the zeroth order thermal energy equation becomes

$$
\frac{2}{7} \frac{1}{x^{2}} \frac{d}{d x}\left(x^{2} \frac{d y^{7 / 2}}{d x}\right)=\left(\frac{r_{\text {cool }}}{\Lambda_{\mathrm{F}, \mathrm{c}}}\right)^{2} x^{-2 \alpha},
$$

where $\left.\Lambda_{\mathrm{F}, \mathrm{c}}=\left[\kappa\left(T_{h}\right) T_{h} / n_{0}^{2} \Lambda\right)\right]^{1 / 2}$ is the Field length, evaluated at the cooling radius (Begelman and McKee 1990; Field 1965). Near $T \sim 2 \times 10^{7} \mathrm{~K}$, the cooling function is nearly independent of $T$. The Field length is then

$$
\Lambda_{\mathrm{F}, \mathrm{c}} \approx 100 \mathrm{kpc}\left(\frac{0.003 \mathrm{~cm}^{-3}}{n}\right)\left(\frac{T_{h}}{2 \times 10^{7} \mathrm{~K}}\right)^{7 / 4},
$$


where we have used the cooling function of McKee and Cowie (1977). The solution of equation $(13 b)$ is then

$$
T(r)=T_{h}\left[\frac{7}{4}\left(\frac{r_{\mathrm{cool}}}{\Lambda_{\mathrm{F}, \mathrm{c}}}\right)^{2} \frac{1}{(2 \alpha-3)(\alpha-1)}\left(\frac{1}{x^{2 \alpha-2}}-\frac{1}{x}\right)+\frac{1}{x}+y_{\infty}^{7 / 2}\left(1-\frac{1}{x}\right)\right]^{2 / 7}
$$

for $\alpha \neq 3 / 2$ and $\alpha \neq 1$;

$$
T(r)=T_{h}\left[-\frac{7}{2}\left(\frac{r_{\mathrm{cool}}}{\Lambda_{\mathrm{F}, \mathrm{c}}}\right)^{2} \frac{\log x}{x}+\frac{1}{x}+y_{\infty}^{7 / 2}\left(1-\frac{1}{x}\right)\right]^{2 / 7}
$$

for $\alpha=3 / 2$, and

$$
T(r)=T_{h}\left[\frac{7}{2}\left(\frac{r_{\mathrm{cool}}}{\Lambda_{\mathrm{F}, \mathrm{c}}}\right)^{2} \log x+\frac{1}{x}+y_{\infty}^{7 / 2}\left(1-\frac{1}{x}\right)\right]^{2 / 7}
$$

for $\alpha=1$. Here, $y_{\infty}$ is an integration constant.

The solution given in equation (15) is a gradually decreasing temperature profile toward the center. A typical cooling flow will have a cooling radius of $\sim 100 \mathrm{kpc}$ and $T_{h} \sim 5 \times 10^{7} \mathrm{~K}$, giving $r_{\text {cool }} / \Lambda_{\mathrm{F}, \mathrm{c}} \sim 0.3$. For the simple, observationally favored case of $\alpha \approx 1$ and $y_{\infty} \approx 1$, we find that the temperature at $r \sim 0.1 r_{\text {cool }}$ will be below its value at the cooling radius by a factor of $\sim(1-0.3 \log 10)^{2 / 7} \approx 0.7$, consistent with $A S C A$ measurements for the hot phase (Fukazawa et al. 1994).

A strong argument in favor of high mass drop-out rates is the high luminosities measured in the low-temperature X-ray emission lines, such as the Fe L lines (Mushotzky et al. 1981; Fabian et al. 1994). These lines demonstrate the presence of low temperature gas that is cooling at a high rate, and buttress the argument for a correspondingly high rate of mass condensation out of the flow. We next show how recycling may significantly alter this point of view.

Consider two phases in pressure equilibrium, a hot phase and a cold phase at respective temperatures $T_{h}$ and $T_{c}$, with number densities $n_{h}$ and $n_{c}$. The cold phase arises from thermally unstable gas suspended in magnetic flux loops. Let $f_{c}$ denote the volume filling factor of the cold phase. The mass $M_{c}$ in cold phase is related to that in the hot, $M_{h}$, by 


$$
M_{c}=\frac{f_{c}}{1-f_{c}} \frac{n_{c}}{n_{h}} M_{h} \approx f_{c} \frac{T_{h}}{T_{c}} M_{h}
$$

The mass cooling rate $\dot{M}_{c}$ of the gas in the cold phase is then related to that in the hot, $\dot{M}_{h}$, by

$$
\dot{M}_{c}=f_{c}\left(\frac{T_{h}}{T_{c}}\right)^{3} \frac{\Lambda\left(T_{c}\right)}{\Lambda\left(T_{h}\right)} \dot{M}_{h}
$$

for a cooling function $\Lambda(T)$. In principle, it is possible for the cooling rate inferred from emission lines generated by the cold phase to exceed that inferred from the hot. We will show below, however, that in our model these rates should be comparable, though some variance in the ratio is to be expected.

The crucial point now is that as the flux loops carrying the cold phase sink they will reconnect to the hot flux tubes. Thermal heat conduction carried along the hot flux tubes will then quickly reheat the cold phase gas to $T_{h}$, resulting in a continual recycling of the cold phase material. The actual mass drop-out rate will thus be much less than inferred from the line luminosities. This is possible provided three criteria are satisfied:

I. The cold loops are able to fall at least as far as the mean distance $\lambda_{h}$ between hot flux tubes in a cooling time: $v_{f f} t_{\mathrm{cool}, \mathrm{c}}>\lambda_{h}$;

\section{II: Loops must enter an evaporative phase on reconnecting;}

III. There is a sufficient number of hot flux tubes penetrating the cool phase region to conduct heat inward from the infinite heat bath surrounding the cool phase to power the total radiative losses from both the cool and hot phases.

These conditions may be met as follows:

I. To make a simple estimate assume that for the purposes of argument that the cooling time is of the order of the time for a loop to cross the cooling region. Then for a volume filling factor of the hot phase within the cooling region of $f_{h} \sim 1$, the covering factor of the hot phase is $\Omega_{h} \sim f_{h}^{\frac{2}{3}} \sim 1$. The cool loops will then collide with the hot phase on the order of a cooling time of 
the cool phase.

II. The cool loops must have a characteristic scale less than the Field length $\Lambda_{F}$ when they reconnect to the hot loops. For loops that are initially magnetically isolated and collide early on ( when they have temperatures of order, say, $3 \times 10^{6} \mathrm{~K}$ ) with hot loops they will undergo evaporation in the classical limit and be recycled since

$$
\Lambda_{F}=3 \mathrm{kpc}\left(\frac{0.03 \mathrm{~cm}^{-3}}{n}\right)\left(\frac{T_{h}}{3 \times 10^{7} \mathrm{~K}}\right)^{5 / 2}\left(\frac{T_{c}}{3 \times 10^{6} \mathrm{~K}}\right)^{1.6},
$$

where we have allowed for the radiation of the cooler material using the cooling approximation given in McKee and Cowie (1977).

Evidence for absorption by very cold clouds has been argued for on the basis of the X-ray spectra in some cooling flows, with inferred characteristic cloud sizes of $\lesssim 1$ pc (Fabian 1994). In our model, such clouds will ultimately result from any small inefficiency in reheating. We suggest that these clouds are shortlived, and will evaporate when they reconnect with hotter loops. For the small cloud sizes suggested, heat conduction occurs in the saturated limit, so that the clouds will disappear in an evaporative wind (McKee and Cowie 1977). The timescale for the clouds to evaporate is

$$
t_{e v}=10^{8} \mathrm{yr}\left(\frac{10 K}{T_{c}}\right)\left(\frac{T_{h}}{3 \times 10^{7} \mathrm{~K}}\right)^{1 / 2} \phi_{s}\left(\frac{\sigma_{0}}{10^{3}}\right)^{3 / 8}\left(\frac{R}{1 \mathrm{pc}}\right)
$$

where $R$ is the cloud radius, and the saturation parameter, $\sigma_{0}=4\left(T_{h} / 3 \times 10^{7} \mathrm{~K}\right)^{2} /\left(n_{h} R_{\mathrm{pc}} \phi_{s}\right)$, is $\sim 10^{3}$, taking $\phi_{s} \sim 0.3$ (Balbus and McKee 1982).

From analyses of absorption-line studies in both X-ray and radio bands the temperature of the clouds must be less than $10 \mathrm{~K}$ (Crawford and Fabian 1992; Dwarakanath et al. 1994). The X-ray absorption measurements indicate H I column densities of the order of $10^{20}-10^{21} \mathrm{~cm}^{-2}$ (Fabian 1994). At the canonical pressure of the central cooling flow this corresponds to scales of only $10^{16}-10^{17} \mathrm{~cm}$ corresponding to cloud evaporation timescales of $\sim 10^{5}-10^{6} \mathrm{yr}$. Therefore, when the reconnection occurs the cold H I will evaporate and be recycled into the hot phase. The cloud destruction time is given by the time for reconnection with hot loops $\left(\sim 10^{8} \mathrm{yr}\right)$. For unit covering factor the cloud destruction rate in the central region of order $R_{\text {central }} \sim 1 \mathrm{kpc}$ is 
$\sim 1\left(R_{\text {central }} / 1 \mathrm{kpc}\right)^{2}\left(N_{\mathrm{HI}} / 10^{21} \mathrm{~cm}^{-2}\right) M_{\odot} \mathrm{yr}^{-1}$

III. The hot phase will occupy most of the volume. A reasonable estimate of the cooling

filling factor is $f_{c} \approx(\Gamma \tau)^{-1}\left(T_{c} / T_{h}\right) \sim 10^{-3}<<1$. From equation (16), only about $1 \%$ of the total intracluster gas within the cooling radius will then be in the cool component. Because of the relatively greater efficiency for generating X-ray photons per unit mass of the cool phase compared to the hot, however, equation (17) shows that the mass cooling rate of the cool component will be comparable to that of the hot, and may even somewhat exceed it. If the cool phase were detected through an X-ray emission line, such a disagreement, which could be interpreted in the standard cooling flow picture as an abundance anomaly of the element producing the line, could equally well be interpreted as evidence for a second, denser and cooler phase.

\section{SUMMARY, CONCLUSIONS, AND OBSERVATIONAL CONSEQUENCES}

We have proposed a model for significantly reducing the mass inflow rates in clusters of galaxies. Reconnection processes acting on the topology of the field regulates the inflow of heat from the vast heat bath of the entire cluster. Reconnection has no energetic advantage at all; it is simply the topology change that allows heating of the matter in cold loops and a relatively fast recycling time. Therefore the cool gas can be used many times, in contrast to conventional cooling flow models in which once the gas cools, it necessarily becomes invisible and can never be recycled. A crucial parameter is the coherence length of the field relative to the cooling radius and the distribution of field energy with scale. When the cooling radius is greater than the field coherence length, cooling flows proceed as usual. When the coherence length is greater than the central cooling radius, however, the thermal energy of the reservoir can be tapped and the mass drop out rates can be very significantly reduced. Three additional conditions must be satisfied: I. Cold loops must be able to fall at least as far as the mean distance between hot loops in a cooling time; II. Loops must enter an evaporative phase on reconnecting; and III. A sufficient number of hot loops must penetrate the cold phase region to power the radiative losses. 
Central regions will be multi-phase with a hot-phase filling factor of order unity and the cold-phase filling factor very much less than unity. There is rapid mass circulation on a timescale of order $10^{8} \mathrm{yr}$.

Filaments will be highly magnetized. In general, the central regions of cooling flows should look very magnetized and filamentary. Detailed rotation measure maps could map the central field geometry. The two-phase model we outline here is consistent with the recent $A S C A$ observations of the centers of cooling flows (Fabian et al. 1994) although many details remain to be understood.

We thank our colleagues for many useful and enjoyable discussions on this subject in particular F. Adams. S. Cowley, M. Donahue, T. Heckman, D. Melrose, R. Mushotsky, R. Sudan and M. Voit. We thank the Institute for Theoretical Physics at UCSB for the stimulating environment where this interdisciplinary effort was possible. The ITP is funded by NSF Grant No. PHY94-07194. A.M. is grateful to the W. Gaertner fund at the University of Chicago for support. 


\section{REFERENCES}

Balbus, S. A., \& McKee, C.F. 1982 ApJ, 252, 529

Begelman, M. C., \& McKee, C. F. 1990 ApJ, 358, 375

Bertshinger, E. 1989 ApJ, 340, 666

Bertschinger, E., \& Meiksin, A. 1986, ApJ, 306, L1

Binney, J., \& Cowie, L. 1981, ApJ, 247, 114

Binney, J., \& Tabor, G. 1995, MNRAS, in press

Chun, E., \& Rosner, R. 1993, ApJ, 408, 678

Crawford, C., \& Fabian, A.C. 1992 MNRAS, 259, 265

Dwarakanath, K. S., van Gorkom, J. H., \& Owen, F. N. 1994 ApJ, 432, 469

Fabian, A. 1994, ARA\&A, 32, 277

Fabian, A. C., Arnaud, K. A., Bautz, M. W., \& Tawara, Y. 1994, ApJ, 436, L63

Field, G.B. 1965 ApJ, 142, 531

Fukazawa, Y. et al. 1994, PASJ, 46, L55

Ge, J.-P. 1991, PhD Thesis, New Mexico Institute of Mining and Technology

Ge, J.-P., \& Owens, F. N. 1993, AJ, 105, 778

Heckman, T., Baum, S. A., van Breugel, W. J., \& McCarthy, P. J. 1989, ApJ, 338, 48

Kulsrud, R. M. 1995, Seminar ITP

McKee, C.F., \& Cowie, L.L. 1977, ApJ, 215, 213

Meiksin, A. 1988, ApJ, 334, 59

Mushotzky, R. F., Holt, S. S., Smith, B. W., Boldt, E., \& Serlemitsos, P. J. 1981, ApJ, 244, L47

Norman, C., Adams, F., Cowley, S., \& Sudan, R. N. 1996, ApJ, submitted 
Pistinner, S., Levinson, A., \& Eichler, D. 1996 (preprint)

Owens, F. N., Eilek, J. A., \& Keel, W. C. 1990, ApJ, 362, 449

Reale, F., Rosner, R., Malagoli, A., Peres, G., \& Serio, S. 1991 MNRAS, 251, 379

Rosner, R., \& Tucker, W. H. 1989, ApJ, 338, 761

Soker, N., \& Sarazin, C. L. 1990, ApJ, 348, 73

Tabor, G., \& Binney, J. 1993, MNRAS, 263, 323

Tao, L. 1996, MNRAS, in press

Taylor, J. B. 1974, Phys. Rev. Lett., 33, 1139

Tribble, P. C. 1989, MNRAS, 238, 1247

Zoabi, E., Soker, N., \& Regev, O. 1996, ApJ (in press) 